\title{
Aspirin in patients admitted to hospital with COVID-19 (RECOVERY): a randomised, controlled, open-label, platform trial
}

RECOVERY Collaborative Group*

\section{Summary}

Background Aspirin has been proposed as a treatment for COVID-19 on the basis of its anti-thrombotic properties. We aimed to evaluate the efficacy and safety of aspirin in patients admitted to hospital with COVID-19.

Methods In this randomised, controlled, open-label, platform trial, several possible treatments were compared with usual care in patients hospitalised with COVID-19. The trial took place at 177 hospitals in the UK, two hospitals in Indonesia, and two hospitals in Nepal. Eligible and consenting adults were randomly allocated in a 1:1 ratio to either usual standard of care plus $150 \mathrm{mg}$ aspirin once per day until discharge or usual standard of care alone using webbased simple (unstratified) randomisation with allocation concealment. The primary outcome was 28 day mortality. All analyses were done by intention to treat. The trial is registered with ISRCTN (50189673) and ClinicalTrials.gov (NCT04381936).

Findings Between Nov 1, 2020, and March 21, 2021, 14892 (66\%) of 22560 patients enrolled into the RECOVERY trial were eligible to be randomly allocated to aspirin. 7351 patients were randomly allocated (1:1) to receive aspirin and 7541 patients to receive usual care alone. Overall, 1222 (17\%) of 7351 patients allocated to aspirin and 1299 (17\%) of 7541 patients allocated to usual care died within 28 days (rate ratio $0 \cdot 96,95 \%$ CI 0 89-1·04; p=0 35). Consistent results were seen in all prespecified subgroups of patients. Patients allocated to aspirin had a slightly shorter duration of hospitalisation (median 8 days, IQR 5 to $>28$, vs 9 days, IQR 5 to $>28$ ) and a higher proportion were discharged from hospital alive within 28 days (75\% vs 74\%; rate ratio 1.06, 95\% CI 1.02-1.10; $\mathrm{p}=0.0062)$. Among patients not on invasive mechanical ventilation at baseline, there was no significant difference in the proportion meeting the composite endpoint of invasive mechanical ventilation or death $(21 \%$ vs $22 \%$; risk ratio $0.96,95 \%$ CI 0.90-1.03; $\mathrm{p}=\mathbf{0} \cdot 23)$. Aspirin use was associated with a reduction in thrombotic events $(4 \cdot 6 \%$ vs $5 \cdot 3 \%$; absolute reduction $0.6 \%$, SE $0.4 \%)$ and an increase in major bleeding events (1.6\% vs $1.0 \%$; absolute increase $0.6 \%$, SE $0 \cdot 2 \%)$.

Interpretation In patients hospitalised with COVID-19, aspirin was not associated with reductions in 28 day mortality or in the risk of progressing to invasive mechanical ventilation or death, but was associated with a small increase in the rate of being discharged alive within 28 days.

Funding UK Research and Innovation (Medical Research Council), National Institute of Health Research, and the Wellcome Trust through the COVID-19 Therapeutics Accelerator.

Copyright (C) 2021 The Author(s). Published by Elsevier Ltd. This is an Open Access article under the CC BY 4.0 license.

\section{Introduction}

Thrombosis is a key feature of severe COVID-19, with 5-30\% of hospitalised patients (depending on illness severity) having a major venous thromboembolic event (mostly pulmonary embolism) and up to $3 \%$ of patients having an arterial thromboembolic event, particularly myocardial infarction and ischaemic stroke., ${ }^{1,2}$ The risk of thromboembolic complications is reported to be higher in COVID-19 than in other acute medical illnesses and viral respiratory infections, and is associated with worse prognosis.,

Anti-platelet therapy might have beneficial effects in severe COVID-19 through several mechanisms, including inhibition of platelet aggregation, reduction of plateletderived inflammation, and blocking of thrombogenic neutrophil extracellular traps. ${ }^{5}$ Aspirin is an affordable, globally available drug which at low doses irreversibly inhibits the cyclooxygenase-1 enzyme, which is responsible for production of thromboxane A2 and proinflammatory prostaglandins. Aspirin can reduce both arterial and venous thrombotic events and has been shown to prevent in-vitro hyperactivity in platelets from patients with SARS-CoV-2. ${ }^{67}$ Existing evidence from randomised trials has shown that $75-150 \mathrm{mg}$ aspirin per day is as effective as higher doses in preventing cardiovascular events. ${ }^{6}$

Seven clinical trials of aspirin in COVID-19 are registered, but none have yet reported on the effect of aspirin therapy in COVID-19. Here we report the results of a large randomised controlled trial of aspirin in patients hospitalised with COVID-19.

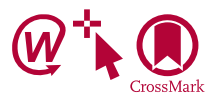

Published Online November 17, 2021 https://doi.org/10.1016/ S0140-6736(21)01825-0

See Online/Comment https://doi.org/10.1016/ S0140-6736(21)01906-1

*The writing committee and trial steering committee are listed at the end of this manuscript and a complete list of the members of the Randomised Evaluation of COVID-19 Therapy (RECOVERY) Collaborative Group is provided in the appendix

Correspondence to: Prof Peter W Horby and Prof Martin J Landray, RECOVERY Central Coordinating Office, Oxford OX3 7LF, UK recoverytrial@ndph.ox.ac.uk See Online for appendix 
Research in context

\section{Evidence before this study}

Patients with COVID-19 are at risk of thromboembolic complications. Anti-thrombotic therapies such as aspirin might be useful to prevent vascular events and improve outcomes. We searched MEDLINE, Embase, bioRxiv, medRxiv, and the WHO International Clinical Trials Registry Platform (ICTRP), from Sept 1, 2019, up to Feb 25, 2021, for completed published randomised clinical trials establishing the effect of aspirin in patients with COVID-19. For MEDLINE and Embase, we used the search terms "Coronavirus infections/", "SARS-CoV-2.mp.", "Coronavirus/" or "CORONAVIRUS.mp", "COVID.mp.", "COVID-19.mp.", "2019-nCoV.mp.", "COVID19.mp", "SARSCoV2.mp", or "SARS-Cov2.mp" and "aspirin.mp", "aspirin/", or "acetylsalicylic acid/", filtered by randomised controlled trials according to validated filters. For medRxiv and bioRxiv, we used the search term "aspirin". We identified no published randomised controlled trials assessing aspirin as a treatment for patients with COVID-19 in any clinical scenario. The WHO ICTRP database listed seven ongoing randomised trials of aspirin, two in outpatients and five in inpatients.
Added value of this study

To the best of our knowledge, the Randomised Evaluation of COVID-19 Therapy (RECOVERY) trial is the first randomised controlled trial to report on the effect of aspirin as a treatment for hospitalised patients with COVID-19. We found that in 14892 adults hospitalised with COVID-19, $150 \mathrm{mg}$ aspirin did not reduce 28 day mortality, and among patients who were not receiving invasive mechanical ventilation at randomisation, did not reduce the probability of progression to the composite outcome of invasive mechanical ventilation or death. Allocation to aspirin was associated with an increase in the rate of being discharged alive within 28 days, but the magnitude of the effect was small ( $1 \%$ absolute difference).

Implications of all the available evidence

Our findings do not support the use of aspirin as a treatment for hospitalised patients with COVID-19.

\section{Methods}

\section{Study design and participants}

The Randomised Evaluation of COVID-19 Therapy (RECOVERY) trial is an investigator-initiated, individually randomised, controlled, open-label, platform trial to evaluate the effects of potential treatments in patients hospitalised with COVID-19. Details of the trial design and results for other treatments evaluated (including lopinavir-ritonavir, hydroxychloroquine, dexamethasone, azithromycin, tocilizumab, convalescent plasma, and colchicine) have been published previously. ${ }^{8-14}$ Aspirin comparison was conducted at 167 hospitals in the UK, two hospitals in Indonesia, and two hospitals in Nepal (appendix pp 5-26), and is supported in the UK by the National Institute for Health Research Clinical Research Network. The trial was coordinated by the Nuffield Department of Population Health at the University of Oxford (Oxford, UK), the trial sponsor. The trial was done in accordance with the principles of the International Conference on Harmonisation Good Clinical Practice guidelines and approved by the UK Medicines and Healthcare products Regulatory Agency and the Cambridge East Research Ethics Committee (reference 20/EE/0101). The protocol, statistical analysis plan, and additional information are available on the study website.

Patients admitted to hospital were eligible for the trial if they had clinically suspected or laboratory-confirmed SARS-CoV-2 infection and no medical history that might, in the opinion of the attending clinician, put the patient at substantial risk if they were to participate in the trial. Children younger than 18 years were not eligible for random assignment to aspirin. Patients with known hypersensitivity to aspirin, a recent history of major bleeding, or currently receiving aspirin or another antiplatelet treatment were excluded. Written informed consent was obtained from all patients, or a legal representative if they were too unwell or unable to provide consent.

\section{Randomisation and masking}

Baseline data were collected using a web-based casereport form that included demographics, amount of respiratory support, major comorbidities, suitability of the study treatment for a particular patient, and treatment availability at the study site (appendix pp 33-34). Eligible and consenting adult patients were assigned in a 1:1 ratio to either usual standard of care or usual standard of care plus aspirin using web-based simple (unstratified) randomisation with allocation concealed until after randomisation (appendix p 30). For some patients, aspirin was unavailable at the hospital at the time of enrolment or was considered by the managing physician to be either definitely indicated or definitely contraindicated. These patients were excluded from the randomised comparison between usual care plus aspirin and usual care alone.

As a platform trial, and in a factorial design, patients could be simultaneously randomly assigned to the following other treatment groups: azithromycin, colchicine, or dimethyl fumarate versus usual care; convalescent plasma or casirivimab and imdevimab versus usual care; and baricitinib versus usual care (appendix p 30). Until Jan 24, 2021, the trial also allowed a subsequent randomisation for patients with progressive COVID-19 (evidence of hypoxia and a hyperinflammatory 
state) to tocilizumab versus usual care. Participants and local study staff were not masked to the allocated treatment. The trial steering committee, investigators, and all other individuals involved in the trial were masked to aggregated outcome data during the trial.

\section{Procedures}

Patients allocated to aspirin received $150 \mathrm{mg}$ by mouth (or nasogastric tube) or by rectum every day until discharge. The $150 \mathrm{mg}$ dose of aspirin once per day was chosen to ensure sufficient inhibition of platelet cyclooxygenase-1 activity in all participants, including those who were overweight. ${ }^{15}$

A single online follow-up form was completed when participants were discharged, had died, or 28 days after randomisation, whichever occurred earliest (appendix pp 35-41). We recorded information on adherence to allocated study treatment, receipt of other COVID-19 treatments, duration of admission, receipt of respiratory or renal support, and vital status (including cause of death). In addition, in the UK, we obtained routine health-care and registry data, including information on vital status (with date and cause of death), discharge from hospital, receipt of respiratory support, or renal replacement therapy.

\section{Outcomes}

Outcomes were assessed 28 days after randomisation, with further analyses specified at 6 months. The primary outcome was all-cause mortality. Secondary outcomes were time to discharge from hospital, and, among patients not on invasive mechanical ventilation at randomisation, progression to invasive mechanical ventilation (including extracorporeal membrane oxygenation) or death. Prespecified subsidiary clinical outcomes were use of non-invasive respiratory support, time to successful cessation of invasive mechanical ventilation (defined as cessation of invasive mechanical ventilation within, and survival to, 28 days), use of renal dialysis or haemofiltration, cause-specific mortality, major bleeding events (defined as intracranial bleeding or bleeding requiring transfusion, endoscopy, surgery, or vasoactive drugs), thrombotic events (defined as acute pulmonary embolism, deep-vein thrombosis, ischaemic stroke, myocardial infarction, or systemic arterial embolism) and major cardiac arrhythmias. Information on suspected serious adverse reactions was collected in an expedited fashion to comply with regulatory requirements.

\section{Statistical analysis}

We did an intention-to-treat comparison between patients randomly assigned to aspirin and patients randomly assigned to usual care but for whom aspirin was both available and suitable as a treatment. For the primary outcome of 28 day mortality, the observed log rank minus the expected statistic and its variance were used to both test the null hypothesis of equal survival curves (ie, the log-rank test) and to calculate the one-step estimate of the average mortality rate ratio. We constructed Kaplan-Meier survival curves to display cumulative mortality over the 28 day period. We used the same method to analyse time to hospital discharge and successful cessation of invasive mechanical ventilation, with patients who died in hospital censored on day 29. Median time to discharge was derived from Kaplan-Meier estimates. For the prespecified composite secondary outcome of progression to invasive mechanical ventilation or death within 28 days (among those not receiving invasive mechanical ventilation at randomisation), and the subsidiary clinical outcomes of receipt of ventilation and use of haemodialysis or haemofiltration, the precise dates were not available, and so the risk ratio was estimated instead.

Prespecified subgroup analyses (defined by characteristics at randomisation, including age, sex, ethnicity, amount of respiratory support, days since symptom onset, and use of corticosteroids) were done for the primary outcome. We did a sensitivity analysis restricting analysis of the primary outcome to patients with a positive PCR test for SARS-COV-2. In addition, we did post-hoc exploratory analyses of the primary and secondary outcomes by venous thromboprophylaxis treatment at randomisation. Observed effects within subgroup categories were compared using a $\chi^{2}$ test for heterogeneity or trend, in accordance with the prespecified analysis plan (appendix p 99).

Estimates of rate and risk ratios are shown with 95\% CIs. All p values are two sided and are shown without adjustment for multiple testing. The full database is held by the study team that collected the data from the study sites and did the analyses at the Nuffield Department of Population Health, University of Oxford (Oxford, UK).

As stated in the protocol, appropriate sample sizes could not be estimated when the trial was being planned at the start of the COVID-19 pandemic (appendix p 33). As the trial progressed, the trial steering committee, whose members were unaware of the results of the trial comparisons, established that sufficient patients should be enrolled to provide at least $90 \%$ power at a two-sided significance level of $1 \%$ to detect a clinically relevant proportional reduction in 28 day mortality of $12.5 \%$ between the two groups. Consequently, on March 21, 2021, the steering committee, masked to the results, closed recruitment to the aspirin comparison as sufficient patients had been recruited.

Analyses were done using SAS version 9.4 and $\mathrm{R}$ version $4 \cdot 0 \cdot 3$. The trial is registered with ISRCTN (50189673) and ClinicalTrials.gov (NCT04381936).

\section{Role of the funding source}

The funders of the study had no role in study design, data collection, data analysis, data interpretation, or writing of the report. 


\section{Results}

Between Nov 1, 2020, and March 21, 2021, 14892 (66\%) of 22560 patients enrolled into the RECOVERY trial were eligible to be randomly allocated to aspirin (ie, aspirin was available in the hospital at the time and the attending clinician was of the opinion that the patient had no known indication for or contraindication to aspirin; figure 1). Baseline characteristics of the patients are presentend (table 1). 7351 patients were randomly allocated to usual care plus aspirin and 7541 were randomly allocated to usual care alone. The mean age of study participants in this comparison was $59 \cdot 2$ years (SD 14.2) and the median time since symptom onset was 9 days (IQR 6-12 days; appendix p 45). At randomisation, 5035 (34\%) patients were receiving thromboprophylaxis with higher-dose low molecular weight heparin (LMWH), $8878(60 \%)$ patients were receiving standard-dose LMWH, and 979 (7\%) patients were not receiving thromboprophylaxis (appendix p 54).

The follow-up form was completed for 7290 (99\%) of 7351 participants in the aspirin group and 7457 (99\%) of 7541 participants in the usual care group. Among participants with a completed follow-up form, 6587 (90\%) patients allocated to aspirin received at least one dose of aspirin and 210 (3\%) allocated to usual care received at

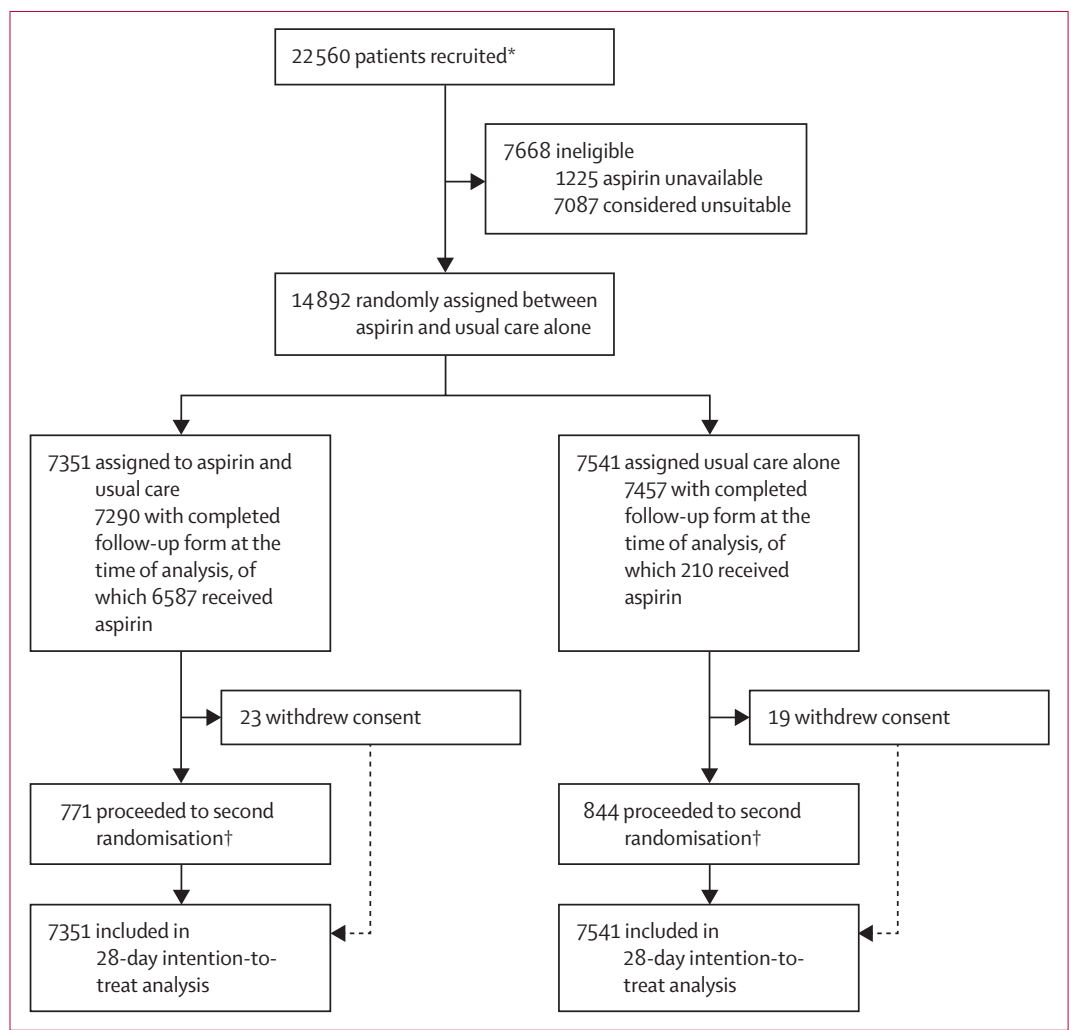

Figure 1: Trial profile

Aspirin unavailable and aspirin unsuitable groups are not mutually exclusive. *Number recruited overall during the period that adult participants could be recruited into the aspirin comparison. Includes 379 (5\%) of 7351 patients in the aspirin group and 407 (5\%) of 7541 patients in the usual care group allocated to tocilizumab. least one dose of aspirin (figure 1; appendix p 46). Of the 6587 participants allocated to aspirin that received at least one dose of aspirin, 5040 (77\%) received aspirin on most days following randomisation $(\geq 90 \%$ of the days from randomisation to time to discharge or 28 days after

\begin{tabular}{|c|c|c|}
\hline & \multicolumn{2}{|c|}{ Treatment allocation } \\
\hline & $\begin{array}{l}\text { Aspirin } \\
(n=7351)\end{array}$ & $\begin{array}{l}\text { Usual care } \\
(\mathrm{n}=7541)\end{array}$ \\
\hline Age (years) & $59 \cdot 2(14 \cdot 1)$ & $59 \cdot 3(14 \cdot 3)$ \\
\hline$<70$ & $5658(77 \%)$ & $5786(77 \%)$ \\
\hline $70-79$ & $1163(16 \%)$ & $1165(15 \%)$ \\
\hline$\geq 80$ & $530(7 \%)$ & $590(8 \%)$ \\
\hline \multicolumn{3}{|l|}{ Sex } \\
\hline Male & $4570(62 \%)$ & $4631(61 \%)$ \\
\hline Female* $^{*}$ & $2781(38 \%)$ & $2910(39 \%)$ \\
\hline \multicolumn{3}{|l|}{ Ethnicity } \\
\hline White & $5474(74 \%)$ & $5655(75 \%)$ \\
\hline $\begin{array}{l}\text { Black, Asian, and minority } \\
\text { ethnic }\end{array}$ & $1176(16 \%)$ & $1202(16 \%)$ \\
\hline Unknown & $701(10 \%)$ & $684(9 \%)$ \\
\hline $\begin{array}{l}\text { Number of days since symptom } \\
\text { onset }\end{array}$ & $9(7-12)$ & $9(6-12)$ \\
\hline $\begin{array}{l}\text { Number of days since } \\
\text { hospitalisation }\end{array}$ & $1(1-3)$ & $2(1-3)$ \\
\hline \multicolumn{3}{|l|}{ Respiratory support received } \\
\hline None or simple oxygen & $4936(67 \%)$ & $5036(67 \%)$ \\
\hline Non-invasive ventilation & $2057(28 \%)$ & $2133(28 \%)$ \\
\hline $\begin{array}{l}\text { Invasive mechanical } \\
\text { ventilation }\end{array}$ & $358(5 \%)$ & $372(5 \%)$ \\
\hline \multicolumn{3}{|l|}{ Biochemistry } \\
\hline C-reactive protein, mg/L & $88(47-146)$ & $91(47-150)$ \\
\hline Creatinine, $\mu \mathrm{mol} / \mathrm{L}$ & $76(63-93)$ & $76(62-92)$ \\
\hline D-dimer, ng/mL & $475(205-1088)$ & $489(210-1083)$ \\
\hline \multicolumn{3}{|l|}{ Previous diseases } \\
\hline Diabetes & $1588(22 \%)$ & $1659(22 \%)$ \\
\hline Heart disease & $776(11 \%)$ & $788(10 \%)$ \\
\hline Chronic lung disease & $1425(19 \%)$ & $1411(19 \%)$ \\
\hline Tuberculosis & $20(<1 \%)$ & $21(<1 \%)$ \\
\hline HIV & $25(<1 \%)$ & $21(<1 \%)$ \\
\hline Severe liver disease $†$ & $67(1 \%)$ & $53(1 \%)$ \\
\hline Severe kidney impairment $\ddagger$ & $214(3 \%)$ & $251(3 \%)$ \\
\hline Any of the previous diseases & $3154(43 \%)$ & $3247(43 \%)$ \\
\hline \multicolumn{3}{|l|}{ Use of corticosteroids } \\
\hline Yes & $6906(94 \%)$ & $7109(94 \%)$ \\
\hline No & $441(6 \%)$ & $425(6 \%)$ \\
\hline Data missing & $4(<1 \%)$ & $7(<1 \%)$ \\
\hline \multicolumn{3}{|l|}{ SARS-CoV-2 test result } \\
\hline Positive & $7140(97 \%)$ & $7327(97 \%)$ \\
\hline Negative & $87(1 \%)$ & $86(1 \%)$ \\
\hline Unknown & $124(2 \%)$ & $128(2 \%)$ \\
\hline \multicolumn{3}{|c|}{$\begin{array}{l}\text { Data are } \mathrm{n}(\%) \text {, mean }(\mathrm{SD}) \text {, or median (IQR). *Includes } 58 \text { pregnant women. } \\
\text { †Defined as requiring ongoing specialist care. } \neq \text { Defined as estimated glomerular } \\
\text { filtration rate lower than } 30 \mathrm{~mL} / \mathrm{min} \text { per } 1.73 \mathrm{~m}^{2} \text {. }\end{array}$} \\
\hline
\end{tabular}


randomisation, whichever was earlier). Use of other treatments for COVID-19 was similar among participants allocated to aspirin and among those allocated to usual care, with nearly $90 \%$ receiving a corticosteroid, about a quarter receiving remdesivir, and an eighth receiving tocilizumab (appendix p 46).

Primary and secondary outcome data were known for $99 \%$ of randomly assigned patients. We observed no significant difference in the proportion of patients who met the primary outcome of 28 day mortality between the two randomised groups (1222 [17\%] of 7351 patients in the aspirin group vs 1299 [17\%] of 7541 patients in the usual care group; rate ratio $0 \cdot 96,95 \%$ CI $0 \cdot 89-1 \cdot 04$; $\mathrm{p}=0.35$; figure 2 , table 2 ). The rate ratio was similar across all prespecified subgroups (figure 3). In an exploratory analysis restricted to the 14467 (97\%) of 14892 patients with a positive SARS-CoV-2 test result, the result was virtually identical (rate ratio 0.96, 95\% CI $0 \cdot 89-1 \cdot 04 ; \mathrm{p}=0 \cdot 31$ ).

Allocation to aspirin was associated with a reduction of 1 day in median time until discharge alive from hospital compared with usual care and an increased rate of discharge alive within 28 days (table 2). Among patients not on invasive mechanical ventilation at baseline, the number of patients progressing to the prespecified composite secondary outcome of invasive mechanical ventilation or death among those allocated to aspirin was similar to that among those allocated to usual care (table 2). There was no evidence that the effect of allocation to aspirin versus usual care on time until discharge alive from hospital, or invasive mechanical ventilation or death differed between the prespecified subgroups of patients (appendix pp 52-53). In a post-hoc exploratory analysis, there was no evidence that the effect of allocation to aspirin versus usual care on the primary and secondary outcomes differed by use of LMWH at randomisation (appendix p 54).

We found no significant differences in the prespecified subsidiary clinical outcomes of causespecific mortality (appendix p 47), use of ventilation, successful cessation of invasive mechanical ventilation, or receipt of renal dialysis or haemofiltration (table 2). As expected with the use of aspirin, the incidence of thrombotic events was lower $(4.6 \%$ vs $5 \cdot 3 \%$, absolute difference $-0 \cdot 6 \%$, SE $0 \cdot 4 \%$ ) and the incidence of major bleeding events was higher $(1.6 \%$ vs $1.0 \%$, absolute difference $0.6 \%$, SE $0.2 \%$ ) in the aspirin group (appendix $\mathrm{p}$ 48) than in the usual care group. The incidence of new cardiac arrhythmias was similar in the two groups (appendix p 49). There were 18 reports of a serious adverse event believed to be related to aspirin, all of which were due to haemorrhagic events (appendix p 50).

\section{Discussion}

In this large, randomised trial involving more than 14000 patients and more than 2000 deaths, allocation to

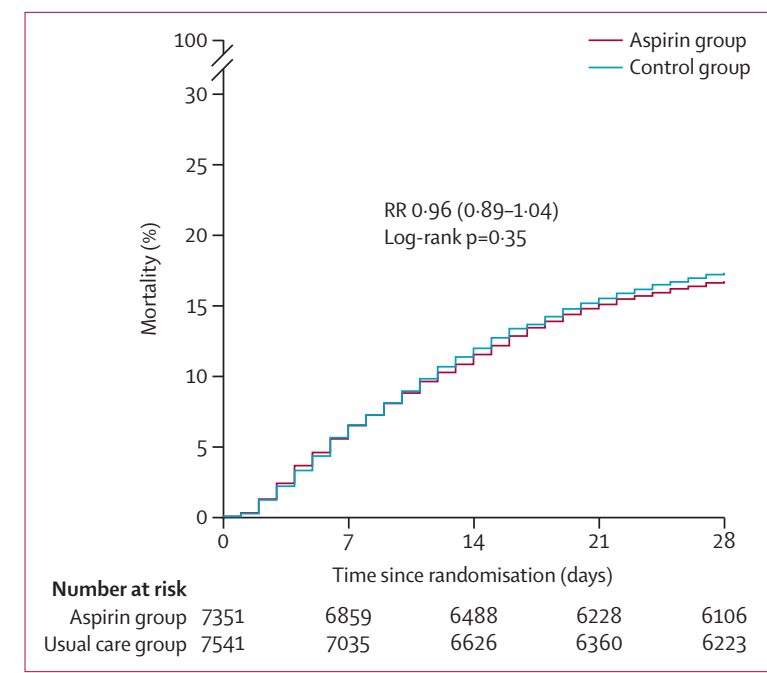

Figure 2: Effect of allocation to aspirin on 28 day mortality $\mathrm{RR}=$ rate ratio.

\begin{tabular}{|c|c|c|c|c|}
\hline & \multicolumn{2}{|c|}{ Treatment allocation } & \multirow[t]{2}{*}{$\mathrm{RR}(95 \% \mathrm{Cl})$} & \multirow[t]{2}{*}{$\mathrm{p}$ value } \\
\hline & Aspirin ( $n=7351)$ & Usual care $(n=7541)$ & & \\
\hline \multicolumn{5}{|l|}{ Primary outcome } \\
\hline 28 day mortality & $1222(17 \%)$ & $1299(17 \%)$ & $0.96(0.89-1.04)$ & 0.35 \\
\hline \multicolumn{5}{|l|}{ Secondary outcomes } \\
\hline $\begin{array}{l}\text { Median time to being discharged } \\
\text { alive (IQR), days }\end{array}$ & $8(5$ to $>28)$ & $9(5$ to $>28)$ &.. &.. \\
\hline $\begin{array}{l}\text { Discharged from hospital within } \\
28 \text { days }\end{array}$ & $5496(75 \%)$ & $5548(74 \%)$ & $1.06(1.02-1.10)$ & 0.0062 \\
\hline $\begin{array}{l}\text { Receipt of invasive mechanical } \\
\text { ventilation or death*}\end{array}$ & 1473/6993 (21\%) & $1569 / 7169(22 \%)$ & $0.96(0.90-1.03)$ & $0 \cdot 23$ \\
\hline Invasive mechanical ventilation & 772/6993 (11\%) & $829 / 7169(12 \%)$ & $0.95(0.87-1.05)$ & $0 \cdot 32$ \\
\hline Death & 1076/6993 (15\%) & $1141 / 7169(16 \%)$ & $0.97(0.90-1.04)$ & 0.39 \\
\hline \multicolumn{5}{|l|}{ Subsidiary clinical outcomes } \\
\hline Use of ventilation & $1131 / 4936(23 \%)$ & $1198 / 5036(24 \%)$ & $0.96(0.90-1.03)$ & $0 \cdot 30$ \\
\hline Non-invasive ventilation & $1101 / 4936(22 \%)$ & $1162 / 5036(23 \%)$ & $0.97(0.90-1.04)$ & 0.36 \\
\hline Invasive mechanical ventilation & $296 / 4936(6 \%)$ & $325 / 5036(6 \%)$ & $0.93(0.80-1.08)$ & 0.35 \\
\hline $\begin{array}{l}\text { Successful cessation of invasive } \\
\text { mechanical ventilation }\end{array}$ & $135 / 358(38 \%)$ & $135 / 372(36 \%)$ & $1.08(0.85-1 \cdot 37)$ & 0.54 \\
\hline Renal replacement therapy & 273/7291 (4\%) & $282 / 7480(4 \%)$ & $0.99(0.84-1.17)$ & 0.93 \\
\hline \multicolumn{5}{|c|}{$\begin{array}{l}\text { RR=rate ratio for the outcomes of } 28 \text {-day mortality and hospital discharge, and rate ratio for the outcome of receipt of } \\
\text { invasive mechanical ventilation or death (and its subcomponents). *Analyses exclude those on invasive mechanical } \\
\text { ventilation at randomisation. }\end{array}$} \\
\hline
\end{tabular}

aspirin was not associated with reductions in mortality or, among patients not on invasive mechanical ventilation at baseline, the risk of progressing to the composite endpoint of invasive mechanical ventilation or death. Allocation to aspirin was, however, associated with a small increase in the rate of being discharged from hospital alive within 28 days. These results were consistent across the prespecified subgroups of age, sex, ethnicity, duration of symptoms before randomisation, amount of respiratory support at randomisation, and use of corticosteroids. 


\begin{tabular}{|c|c|c|c|c|c|}
\hline & Aspirin group & Usual care group & & & $\operatorname{RR}(95 \% \mathrm{Cl})$ \\
\hline \multicolumn{6}{|l|}{ Age $\left(\chi_{1}^{2}=2 \cdot 0 ; p=0 \cdot 15\right)$} \\
\hline$<70$ years & $578 / 5658(10 \%)$ & 619/5786 (11\%) & $\rightarrow$ & - & $0.95(0.85-1.07)$ \\
\hline 70-79 years & 376/1163 (32\%) & 401/1165 (34\%) & $\longrightarrow$ & - & $0.94(0.81-1.08)$ \\
\hline$\geq 80$ & $268 / 530(51 \%)$ & $279 / 590(47 \%)$ & & $\longrightarrow$ & $1.13(0.95-1.34)$ \\
\hline \multicolumn{6}{|l|}{$\operatorname{Sex}\left(\chi_{1}^{2}=2 \cdot 1 ; p=0 \cdot 15\right)$} \\
\hline Male & $763 / 4570(17 \%)$ & $833 / 4631(18 \%)$ & $\rightarrow$ & & $0.92(0.83-1.02)$ \\
\hline Female & $459 / 2781(17 \%)$ & $466 / 2910(16 \%)$ & -1 & $=$ & $1.04(0.91-1.18)$ \\
\hline \multicolumn{6}{|l|}{ Ethnicity $\left(\chi_{1}^{2}=0.0 ; p=0.96\right)$} \\
\hline White & 943/5474 (17\%) & $1023 / 5655(18 \%)$ & $\rightarrow$ & & $0.95(0.87-1.04)$ \\
\hline Black, Asian, and minority ethnic & 197/1176 (17\%) & $212 / 1202(18 \%)$ & $\rightarrow-1$ & — & $0.94(0.78-1.15)$ \\
\hline \multicolumn{6}{|c|}{ Time since symptom onset $\left(\chi_{1}^{2}=0.8 ; p=0.37\right)$} \\
\hline$\leq 7$ days & $493 / 2424(20 \%)$ & $563 / 2581(22 \%)$ & $\rightarrow$ & - & $0.93(0.82-1.05)$ \\
\hline$>7$ days & $729 / 4923(15 \%)$ & $735 / 4954(15 \%)$ & $\rightarrow$ & $\vdash$ & $1.00(0.90-1.11)$ \\
\hline \multicolumn{6}{|c|}{ Respiratory support at randomisation $\left(\chi_{1}^{2}=0.3 ; p=0.60\right)$} \\
\hline None or simple oxygen & $537 / 4936(11 \%)$ & $549 / 5036(11 \%)$ & $\rightarrow$ & - & $1.00(0.89-1.13)$ \\
\hline Non-invasive ventilation & $539 / 2057(26 \%)$ & $592 / 2133(28 \%)$ & $\rightarrow$ & & $0.93(0.83-1.05)$ \\
\hline Invasive mechanical ventilation & $146 / 358(41 \%)$ & $158 / 372(43 \%)$ & $\longrightarrow$ & - & $0.97(0.77-1.22)$ \\
\hline \multicolumn{6}{|c|}{ Use of corticosteroids $\left(\chi_{1}^{2}=0.6 ; p=0 \cdot 42\right)$} \\
\hline Yes & $1141 / 6906(17 \%)$ & $1227 / 7109(17 \%)$ & $\rightarrow$ & & $0.95(0.88-1.03)$ \\
\hline No & $79 / 441(18 \%)$ & $71 / 425(17 \%)$ & - & & $1.09(0.79-1.51)$ \\
\hline \multirow[t]{2}{*}{ All participants } & $1222 / 7351(17 \%)$ & $1299 / 7541(17 \%)$ & $<$ & & $\begin{array}{l}0.96(0.89-1.04) \\
p=0.35\end{array}$ \\
\hline & & & 0.75 & $1 \cdot 5$ & \\
\hline
\end{tabular}

Figure 3: Effect of allocation to aspirin on 28 day mortality by baseline characteristics

Subgroup-specific rate ratio estimates are represented by squares (with areas of the squares proportional to the amount of statistical information) and the lines through them correspond to $95 \% \mathrm{Cls}$. The ethnicity, days since onset, and use of corticosteroids subgroups exclude patients with missing data, but these patients are included in the overall summary diamond. $\mathrm{RR}=$ rate ratio.

As expected, allocation to aspirin was associated with an increased risk of major bleeding and a decreased risk of thromboembolic complications, such that for every 1000 patients treated with aspirin, approximately six more patients would have a major bleeding event and approximately six fewer patients would have a thromboembolic event. The rate of reported thromboembolic events in our study population was low $(5 \cdot 3 \%$ in the usual care group) in comparison with previous reports. ${ }^{1,2}$ This finding could be because participants were not systematically screened for thromboembolic events, could be related to the widespread use of corticosteroids in the trial population, resulting in reduced thromboinflammatory stimulus, or could be because of the exclusion of patients already receiving aspirin due to previous cardiovascular disease. It is possible that aspirin might have a more meaningful benefit in populations with a higher thrombotic risk, although there would also probably be a corresponding increase in bleeding risk. ${ }^{16}$

The pathogenesis of thromboembolism in COVID-19 is likely to be multifactorial. Coagulopathy is common in severe COVID-19 and is associated with an inflammatory state, neutrophil extracellular traps, and poor outcomes. ${ }^{2,17-21}$ Platelet activation is increased as a result (and potentially by direct interaction with the virus), amplifying inflammation locally and triggering immunothrombosis. ${ }^{22,23}$ In addition, SARS-CoV-2 infection can cause inflammation, dysfunction, and disruption of the vascular endothelium in multiple organs, potentially via direct entry through the angiotensin-converting enzyme 2 receptor. ${ }^{24-26}$ The resulting endothelial injury and tissue factor exposure promote thrombosis in the pulmonary circulation and other vascular beds, with microangiopathy and alveolar capillary occlusion contributing to the diffuse alveolar damage and hypoxaemia seen in COVID-19.25,27 Furthermore, in autopsy studies pulmonary microthrombi are nine times more frequent in patients with COVID-19 than patients with influenza. ${ }^{25}$

A large number of randomised controlled trials of antithrombotic therapy in COVID-19 are registered, including trials of therapeutic doses of heparin, directacting oral anticoagulants, anti-platelet agents, serine protease inhibitors, and thrombolytics. ${ }^{28}$ In patients who 
are critically ill, the INSPIRATION, REMAP-CAP, ACTIV-4a, and ATTAC trials did not report a benefit in clinical outcomes from therapeutic anticoagulation..$^{29,30}$ Similarly, preliminary results from the COALIZAOACTION trial ${ }^{31}$ did not show a benefit from therapeutic anticoagulation (either heparin or rivaroxaban) in a combined endpoint of mortality, successful discharge, or need for oxygen in hospitalised patients with elevated D-dimers. However, the REMAP-CAP, ACTIV-4a, and ATTAC investigators have reported that in patients not critically ill with COVID-19, compared with thromboprophylaxis doses heparin at therapeutic doses was associated with an absolute increase of $4.6 \%$ (95\% credible interval $0 \cdot 7-8 \cdot 1)$ in the proportion of participants surviving to hospital discharge without receipt of organ support during the first 21 days. ${ }^{32}$

Although there are no other published randomised trial data on the use of aspirin in COVID-19, the REMAP-CAP, ACTIV-4a, and ATTAC report does suggest that antithrombotic therapy might be important in some patients. ${ }^{32}$ The absence of meaningful benefit from aspirin in our trial could be because anti-platelet therapy confers no clinically significant additional benefit on top of high rates of anti-thrombotic therapy with LMWH and corticosteroid treatment diminishing thromboinflammatory stimulation. Alternatively, other non-platelet pathways leading to thrombosis and alveolar damage might be more important determinants of clinical outcomes.

Any potential benefit of antithrombotic therapies in patients with COVID-19 could also depends on timing of treatment initiation, especially if thrombi have already developed by the time of admission. ${ }^{33}$ Thromboembolic events and microthrombi are common in patients with COVID-19 on either prophylactic or therapeutic anticoagulation. ${ }^{34}$ The apparent absence of benefit in INSPIRATION and the REMAP-CAP, ACTIV-4a, and ATTAC severe disease cohorts suggests that these patients might have passed the point at which any benefit from therapeutic anticoagulation could be gained. ${ }^{29,30}$ Although we found no evidence of heterogeneity on the basis of duration of symptoms, baseline disease severity, or background thrombotic prophylaxis regimen, ongoing trials of aspirin in ambulatory populations and those exploring more potent anti-platelet inhibition and fibrinolysis should provide further insights.

The strengths of this trial included that it was randomised, had a large sample size, broad eligibility criteria, and $99 \%$ of patients were followed up for the primary outcome. The trial also had some limitations. Detailed information on radiological or physiological outcomes was not collected. Although this randomised trial is open label (ie, participants and local hospital staff are aware of the assigned treatment), the primary and secondary outcomes are unambiguous and were ascertained without bias through linkage to routine health records. However, it cannot be excluded that reporting of thromboembolic and bleeding events might have been influenced by knowledge of treatment allocation. Nevertheless, the proportional effects of aspirin on these events were very similar to those reported in previous large clinical trials of aspirin in people with previous cardiovascular disease. ${ }^{6}$

The RECOVERY trial only studied patients with COVID-19 who were hospitalised, and therefore is not able to provide evidence on the safety and efficacy of aspirin used in other patient groups. Further studies to identify the safety and efficacy of aspirin in patients with COVID-19 who are not hospitalised are needed and are ongoing.

In summary, the results of this large, randomised trial do not support the addition of aspirin to standard thromboprophylaxis or therapeutic anticoagulation in patients hospitalised with COVID-19.

\section{Contributors}

This manuscript was initially drafted by PWH and MJL, further developed by the Writing Committee, and approved by all members of the trial steering committee. PWH and MJL approved the data and analyses, and for the fidelity of this report to the study protocol and data analysis plan. PWH, JKB, MHB, LCC, JD, SNF, RLH, TJ, EJ, KJ, WSL, AM, AM, KR, GT, MM, RH, and MJL designed the trial and study protocol. MM, MC, GP-A, LP, NJB, ST, VC, TB, HT, BE, DC, TW, RS, CG, and the Data Linkage team at the RECOVERY Coordinating Centre, and the Health Records and Local Clinical Centre staff listed in the appendix collected the data. NS, ES, and JRE did the statistical analysis. All authors contributed to data interpretation and critical review and revision of the manuscript. PWH and MJL had access to the study data and had final responsibility for the decision to submit for publication.

\section{Data monitoring committee}

Peter Sandercock, Janet Darbyshire, David DeMets, Robert Fowler, David Lalloo, Mohammed Munavvar (from January, 2021), Ian Roberts (until December, 2020), and Janet Wittes.

\section{Declaration of interests}

The authors declare no competing interests or financial relationships relevant to the submitted work. No form of payment was given to anyone to produce the manuscript. The Nuffield Department of Population Health at the University of Oxford has a staff policy of not accepting honoraria or consultancy fees directly or indirectly from industry.

\section{Data sharing}

The protocol, consent form, statistical analysis plan, definition and derivation of clinical characteristics and outcomes, training materials, regulatory documents, and other relevant study materials are available online at https://www.recoverytrial.net. As described in the protocol, the trial Steering Committee will facilitate the use of the study data and approval will not be unreasonably withheld. Deidentified participant data will be made available to researchers registered with an appropriate institution within 3 months of publication. However, the Steering Committee will need to be satisfied that any proposed publication is of high quality, honours the commitments made to the study participants in the consent documentation and ethical approvals, and is compliant with relevant legal and regulatory requirements (eg, relating to data protection and privacy). The Steering Committee will have the right to review and comment on any draft manuscripts before publication. Data will be made available in line with the policy and procedures described at https://www.ndph.ox.ac.uk/data-access. Individuals wishing to request access should complete the form at https://www.ndph.ox.ac.uk/files/ about/data_access_enquiry_form_13_6_2019.docx and e-mail data. access@ndph.ox.ac.uk.

\section{Acknowledgments}

Above all, we would like to thank the thousands of patients who participated in this trial. We would also like to thank the many doctors, nurses, pharmacists, other allied health professionals, and research administrators at the participating hospitals in Nepal and Indonesia 
and at 176 UK National Health Service hospital organisations across the whole of the UK, supported by staff at the National Institute of Health Research (NIHR) Clinical Research Network, NHS DigiTrials, Public Health England, Department of Health and Social Care, the Intensive Care National Audit and Research Centre, Public Health Scotland, National Records Service of Scotland, the Secure Anonymised Information Linkage at the University of Swansea, and the NHS in England, Scotland, Wales, and Northern Ireland. The RECOVERY trial is supported by grants to the University of Oxford from the UK Research and Innovation (UKRI) and the NIHR (grant reference MC_PC_19056), the Department of Health and Social Care (DHSC), UKRI, and NIHR COVID-19 Rapid Response Grant (COV19. RECPLA), and the Wellcome Trust (grant referebce 222406/Z/20/Z) through the COVID-19 Therapeutics Accelerator. Core funding is provided by the NIHR Oxford Biomedical Research Centre, Wellcome, the Bill \& Melinda Gates Foundation, the Foreign, Commonwealth, and Development Department, Health Data Research UK, the Medical Research Council Population Health Research Unit, the NIHR Health Protection Unit in Emerging and Zoonotic Infections, and NIHR Clinical Trials Unit Support Funding. TJ is supported by a grant from the UK Medical Research Council (MC_UU_00002/14) and an NIHR Senior Research Fellowship (NIHR-SRF-2015-08-001). WSL is supported by core funding provided by NIHR Nottingham Biomedical Research Centre. Combiphar supplied colchicine free of charge for use in this trial in Indonesia. AbbVie contributed some supplies of lopinavir-ritonavir for use in this trial. Tocilizumab was provided free of charge for this trial by Roche Products. Casivirimab and imdevimab was provided free of charge for this trial by Regeneron. The views expressed in this publication are our own and not necessarily those of the NHS or the NIHR.

Writing committee (on behalf of the RECOVERY Collaborative Group) Peter W Horby,* Guilherme Pessoa-Amorim, * Natalie Staplin,* Jonathan R Emberson, Mark Campbell, Enti Spata, Leon Peto, Nigel J Brunskill, Simon Tiberi, Victor Chew, Thomas Brown, Hasan Tahir, Beate Ebert, David Chadwick, Tony Whitehouse, Rahuldeb Sarkar, Clive Graham, J Kenneth Baillie, Buddha Basnyat, Maya H Buch, Lucy C Chappell, Jeremy Day, Saul N Faust, Raph L Hamers, Thomas Jaki, Edmund Juszczak, Katie Jeffery, Wei Shen Lim, Alan Montgomery, Andrew Mumford, Kathryn Rowan, Guy Thwaites, Marion Mafham, $\uparrow$ Richard Haynes, $\uparrow$ and Martin J Landray $\uparrow$. *PWH, GP-A, and NS contributed equally. $\dagger \mathrm{MM}, \mathrm{RH}$, and MJL contributed equally.

\section{References}

1 Jimenez D, Garcia-Sanchez A, Rali P, et al. Incidence of VTE and bleeding among hospitalized patients with coronavirus disease 2019: a systematic review and meta-analysis. Chest 2021; 159: 1182-96.

2 Kunutsor SK, Laukkanen JA. Incidence of venous and arterial thromboembolic complications in COVID-19: a systematic review and meta-analysis. Thromb Res 2020; 196: 27-30.

3 Klok FA, Kruip M, van der Meer NJM, et al. Confirmation of the high cumulative incidence of thrombotic complications in critically ill ICU patients with COVID-19: an updated analysis. Thromb Res 2020; 191: 148-50.

4 Smilowitz NR, Subashchandran V, Yuriditsky E, et al. Thrombosis in hospitalized patients with viral respiratory infections versus COVID-19. Am Heart J 2021; 231: 93-95.

5 Carestia A, Davis RP, Grosjean H, Lau MW, Jenne CN. Acetylsalicylic acid inhibits intravascular coagulation during Staphylococcus aureus-induced sepsis in mice. Blood 2020; 135: 1281-86.

6 Antithrombotic Trialists C. Collaborative meta-analysis of randomised trials of antiplatelet therapy for prevention of death, myocardial infarction, and stroke in high risk patients. BMJ 2002 324: 71-86.

7 Manne BK, Denorme F, Middleton EA, et al. Platelet gene expression and function in patients with COVID-19. Blood 2020; 136: 1317-29.

8 RECOVERY Collaborative Group, Horby PW, Mafham M, et al. Lopinavir-ritonavir in patients admitted to hospital with COVID-19 (RECOVERY): a randomised, controlled, open-label, platform trial. Lancet 2020; 396: 1345-52.
9 RECOVERY Collaborative Group, Horby P, Mafham M, et al. Effect of hydroxychloroquine in hospitalized patients with COVID-19. N Engl J Med 2020; 383: 2030-40.

10 RECOVERY Collaborative Group, Horby P, Lim WS, et al. Dexamethasone in hospitalized patients with Covid-19. N Engl J Med 2021; 384: 693-704.

11 RECOVERY Collaborative Group. Azithromycin in patients admitted to hospital with COVID-19 (RECOVERY): a randomised, controlled, open-label, platform trial. Lancet 2021; 397: 605-12.

12 RECOVERY Collaborative Group. Tocilizumab in patients admitted to hospital with COVID-19 (RECOVERY): a randomised, controlled open-label, platform trial. Lancet 2021; 397: 1637-45.

13 RECOVERY Collaborative Group. Convalescent plasma in patients admitted to hospital with COVID-19 (RECOVERY): a randomised controlled, open-label, platform trial. Lancet 2021; published May 21 https://doi.org/10.1016/S0140-6736(21)00897-7.

14 RECOVERY Collaborative Group, Horby PW, Campbell M, et al. Colchicine in patients admitted to hospital with COVID-19 (RECOVERY): a randomised, controlled, open-label, platform trial. medRxiv 2021; published May 18. https://doi.org/10.1101/ 2021.05.18.21257267 (preprint).

15 Rocca B, Fox KAA, Ajjan RA, et al. Antithrombotic therapy and body mass: an expert position paper of the ESC Working Group on Thrombosis. Eur Heart J 2018; 39: 1672-86f.

16 Antithrombotic Trialists C, Baigent C, Blackwell L, et al. Aspirin in the primary and secondary prevention of vascular disease: collaborative meta-analysis of individual participant data from randomised trials. Lancet 2009; 373: 1849-60.

17 Jin S, Jin Y, Xu B, Hong J, Yang X. Prevalence and impact of coagulation dysfunction in COVID-19 in China: a meta-analysis. Thromb Haemost 2020; 120: 1524-35.

18 Zhang A, Leng Y, Zhang Y, et al. Meta-analysis of coagulation parameters associated with disease severity and poor prognosis of COVID-19. Int J Infect Dis 2020; 100: 441-48.

19 Skendros P, Mitsios A, Chrysanthopoulou A, et al. Complement and tissue factor-enriched neutrophil extracellular traps are key drivers in COVID-19 immunothrombosis. J Clin Invest 2020; 130: 6151-57.

20 Middleton EA, He XY, Denorme F, et al. Neutrophil extracellular traps contribute to immunothrombosis in COVID-19 acute respiratory distress syndrome. Blood 2020; 136: 1169-79.

21 Petito E, Falcinelli E, Paliani U, et al. Association of neutrophil activation, more than platelet activation, with thrombotic complications in coronavirus disease 2019. J Infect Dis 2021; 223: 933-44.

22 Taus F, Salvagno G, Cane S, et al. Platelets promote thromboinflammation in SARS-CoV-2 pneumonia. Arterioscler Thromb Vasc Biol 2020; 40: 2975-89.

23 Zhang S, Liu Y, Wang X, et al. SARS-CoV-2 binds platelet ACE2 to enhance thrombosis in COVID-19. I Hematol Oncol 2020; 13: 120

24 Hariri LP, North CM, Shih AR, et al. Lung histopathology in coronavirus disease 2019 as compared with severe acute respiratory sydrome and H1N1 influenza: a systematic review. Chest 2021; 159: 73-84.

25 Ackermann M, Verleden SE, Kuehnel M, et al. Pulmonary vascular endothelialitis, thrombosis, and angiogenesis in covid-19. N Engl J Med 2020; 383: 120-28.

26 Varga Z, Flammer AJ, Steiger P, et al. Endothelial cell infection and endotheliitis in COVID-19. Lancet 2020; 395: 1417-18.

27 Carsana L, Sonzogni A, Nasr A, et al. Pulmonary post-mortem findings in a series of COVID-19 cases from northern Italy: a two-centre descriptive study. Lancet Infect Dis 2020; 20: 1135-40.

28 Talasaz AH, Sadeghipour P, Kakavand H, et al. Recent randomized trials of antithrombotic therapy for patients with COVID-19: JACC state-of-the-art review. J Am Coll Cardiol 2021; 77: 1903-21.

29 The ATTAC, ACTIV-4a and REMAP-CAP Investigators. Therapeutic anticoagulation in critically ill patients with COVID-19: preliminary report. New Engl J Med 2021; 385: 777-89.

30 Inspiration Investigators, Sadeghipour P, Talasaz AH, et al. Effect of intermediate-dose vs standard-dose prophylactic anticoagulation on thrombotic events, extracorporeal membrane oxygenation treatment, or mortality among patients with COVID-19 admitted to the intensive care unit: the INSPIRATION randomized clinical trial. JAMA 2021; 325: 1620-30. 
Articles

31 American College of Cardiology. AntiCoagulaTIon cOroNavirus: ACTION. 2021. https://www.acc.org/Latest-in-Cardiology/ Clinical-Trials/2021/05/14/03/11/ACTION (accessed May 22, 2021).

32 The ATTAC, ACTIV-4a and REMAP-CAP Investigators. Therapeutic anticoagulation in non-critically ill patients with COVID-19. New Engl J Med 2021; 385: 790-802.
33 Yamakawa K, Umemura Y, Murao S, Hayakawa M, Fujimi S. Optimal timing and early intervention with anticoagulant therapy for sepsis-induced disseminated intravascular coagulation. Clin Appl Thromb Hemost 2019; 25: 1076029619835055.

34 Klok FA, Kruip M, van der Meer NJM, et al. Incidence of thrombotic complications in critically ill ICU patients with COVID-19. Thromb Res 2020; 191: 145-47. 